\title{
On the impact of digital music distribution*
}

\author{
Kiho Yoon \\ Department of Economics, Korea University \\ Anam-dong, Sungbuk-gu, Seoul, Korea 136-701 \\ kiho@korea.ac.kr \\ $\underline{\text { http://econ.korea.ac.kr/ kiho }}$ \\ and \\ IIR, Hitotsubashi University \\ 2-1 Naka, Kunitachi, Tokyo 186-8603, Japan \\ k_yoon@iir.hit-u.ac.jp
}

September 2007

\begin{abstract}
We present a framework to evaluate the impact of digital music distribution. We set up a representative model that enables the comparative static analysis. We then interpret two empirical observations about the music industry, the sales decline and the price constancy, and fit the model to these observations. We find that, while the impact of digitalization on the producers' profits is probably negative, it may not be as severe as the observed impact on the quantity. On the other hand, the impact of digitalization on the consumer surplus is unambiguously positive. The impact on the social welfare is rather ambiguous, but numerical calculations reveal that the social welfare has increased for most parameter values. We also show that enhanced copyright protection to counteract the impacts of digitalization may have an unexpected effect on the social welfare. JEL Classification: K11; L86; O34
\end{abstract}

Keywords: Music industry; Digital music; Copyright; File sharing

I thank Sadao Nagaoka and the seminar participants at the IIR workshop for helpful comments. 


\title{
On the impact of digital music distribution
}

\author{
Kiho Yoon
}

\section{Introduction}

The music industry has experienced significant changes in recent years. In particular, the advent of broadband networks made it possible to freely download unauthorized copies of pre-recorded music files via peer-to-peer (P2P) technologies: Consumers can transmit the music in digital format among themselves using the online 'file-sharing' technology provided by Napster, Kazaa, Grokster, and others. These changes have generated heated debates regarding the possible impacts on the sound-recording industry. Some argue that the decline in the sales of recorded music endangers the viability of the industry, while others say that these changes may ultimately benefit the producers.

There exist some empirical works including Michel (2006), Oberholzer and Strumpf (2005), Peitz and Waelbroeck (2004), and Zentner (2006), as well as some theoretical discussion including Liebowitz (2005, 2006) and Peitz and Waelbroeck (2005, 2006b) on the impacts of file-sharing technology. Most of the works, however, are concerned with the causes for the sales decline of pre-recorded music. ${ }^{1}$ In particular, no previous work has studied the welfare consequences.

This paper aims to provide a framework to evaluate the impact of digital music distribution on the social welfare as well as on the profit and the consumer surplus. To do so, we set up a representative model that enables the comparative static analysis before and after the advent of digital distribution technology.

The 'digitalization,' i.e., the distribution of music files in digital format using online technology, affects several aspects of the industry. First, the unauthorized copies shared among end-users create competitive pressure on the legitimate products. That is, the copies are (inferior) substitutes for the original, and so possibly harm the producers of the music by decreasing their sales and profits. On the other hand, it is often argued that the 'sampling,' i.e., consumers' listening to the music before purchasing and finding the better fits for their tastes, afforded by the online technology may in effect boost the demand for the original, and help the producers. Finally, the digitalization may lower the producers' costs of distribution, marketing, and promotion.

It is ultimately an empirical question to determine the net impacts of digitalization. Hence, after setting up a framework to evaluate the impacts, we start with interpreting

\footnotetext{
${ }^{1}$ See Section 3 below for some of their results.
} 
two empirical observations of the music industry. The first and the most obvious observation is the sales decline. Liebowitz (2006, p. 14), for example, estimates that the actual sales in the United States have dropped up to $30 \%$ after digitalization. While various explanations are possible, it is most plausible that the file sharing activity exerted a strong substitution effect. Another interesting and rather puzzling observation is the virtual constancy of price. Liebowitz (2006, p. 21) again observes that 'the list prices adjusted for inflation have been virtually constant for the last decade.' If the unauthorized copies are substitutes, then the price of the legitimate copies should have decreased due to the competitive pressure. That did not happen, however.

Several reasons may be proposed for the price constancy. First, it may be argued that the producers were in a competitive environment to begin with and so could not afford to decrease the price. But this can be easily discarded since the big 4 record labels (Universal, Sony BMG, EMI, Warner) essentially dominate the market and they are sometimes alleged to collude on price fixing. ${ }^{2}$ Alternatively, it is possible that consumers are substantially differentiated with respect to their valuations and attitudes towards the music so that the producers reacted by concentrating on the higher segment of the consumer population. In other words, the digitalization may have made it possible for the producers to provide more value to the high-valuation consumers so it was best to take a price strategy on them (while sacrificing the quantity on the low-valuation consumers.) Our framework supports the latter interpretation. We find that the sampling effect substantially increased consumers' benefits and it worked better for the highvaluation consumers.

We then fit the model to these empirical observations. We first find that, while the impact of digitalization on the producers' profits is probably negative, it may not be as severe as the observed impact on the quantity. In particular, we show that the sampling effect sometimes acts to reduce the revenue while it always increases the profit. Moreover, digitalization may lower the fixed costs of distribution as well as increasing the additional revenue from complementary products such as live performance. On the other hand, the impact of digitalization on the consumer surplus is unambiguously positive: Consumers have benefited from all the possible effects of digitalization. We find that the impact on the social welfare is rather ambiguous. We show that the impact is positive if and only if consumers' reproduction costs were relatively small. Numerical calculations further reveal that the social welfare has increased for most parameter values. We also show that enhanced copyright protection to counteract the impacts of

\footnotetext{
${ }^{2}$ See FTC Press Release on May 10, 2000, 'Record Companies Settle FTC Charges of Restraining Competition in CD Music Market.’
} 
digitalization may have an unexpected effect on the social welfare.

The rest of the paper is organized as follows. Section 2 sets up the model to evaluate the impacts of digitalization, and does some comparative statics before and after digitalization. Section 3 discusses the empirical observations, and determines the possible impacts of digitalization. It also briefly discusses the possible effect of enhanced copyright protection on the social welfare. Section 4 concludes.

\section{The model}

Consider a music product that is produced by a monopolistic producer (record company, music label, or publisher). We first study the benchmark case of traditional distribution. We then see how the digitalization of music distribution affects the relevant variables. We finally discuss the possible effect of further copyright protection. The model we employ is the one developed in Yoon (2002) for the study of copyright protection. Similar models are used in subsequent works including Belleflamme (2003) and Bae and Choi (2006). ${ }^{3}$

\subsection{The benchmark}

There are consumers who are interested in the product. Each consumer consumes at most one unit of the product. We denote the set of consumers by $I$ and the valuation of consumer $i \in I$ by $v_{i}$. Consumers can consume the product either by purchasing from the original producer or by making unauthorized reproductions, i.e., by making copies. When $i \in I$ buys the product, her net utility is $v_{i}-p$, where $p$ is the monopolist's price. When $i \in I$ copies the product, her net utility is $(1-\alpha) v_{i}-z$. The parameter $\alpha$ measures the quality degradation of a copy, with $0<\alpha<1$. $^{4}$ The parameter $z$ captures the reproduction costs, including the physical costs as well as the inconveniences consumers have to bear in making the copies. If we let $w_{i} \equiv \alpha v_{i}+z$ be termed as the gross reproduction cost, the net utility from a copy is $v_{i}-w_{i}$. The utility when a consumer does not consume the product is normalized to zero.

Consumer $i$ will make the following choices depending on the relative magnitudes of $v_{i}, w_{i}$, and $p$. When $p=\min \left\{v_{i}, w_{i}, p\right\}$, consumer $i$ purchases from the producer. When $w_{i}=\min \left\{v_{i}, w_{i}, p\right\}$, consumer $i$ makes an unauthorized copy. When

\footnotetext{
${ }^{3}$ Peitz and Waelbroeck (2006a) is a comprehensive review of the relevant literature.

${ }^{4}$ The quality degradation may also come from the lack of accompanying lyrics, the lack of photographic illustrations of the singers, the lack of technical supports, and so on. See Yoon (2002) for a more detailed discussion of the model setup.
} 
$v_{i}=\min \left\{v_{i}, w_{i}, p\right\}$, consumer $i$ does not consume the product.

For a concrete analysis, assume that consumers' valuations are uniformly distributed over the unit interval $[0,1]$. Thus, we can identify the set $I$ of consumers with the set of valuations, which is the unit interval. Then, consumers' choices given the price can be summarized as follows.

(a) When $p<z /(1-\alpha)$ : This is the case when the monopolist's price is set low enough that no consumer makes an unauthorized copy. Consumers who belong to $[0, p)$ do not consume the product, while consumers who belong to $[p, 1]$ buy from the monopolist.

(b) When $p \geq z /(1-\alpha)$ : This is the case when the unauthorized reproduction exists. Consumers who belong to $[0, z /(1-\alpha))$ do not consume the product, consumers who belong to $[z /(1-\alpha),(p-z) / \alpha)$ make unauthorized copies, and consumers who belong to $[(p-z) / \alpha, 1]$ buy from the monopolist.

This is a result in Yoon (2002, Proposition 2), and can be easily proved by using a diagram similar to Figure 1 below. ${ }^{5}$ By the way, we observe both the legitimate product and its unauthorized copies in most real markets. As a matter of fact, copyright protection and related issues may not have attracted much academic attention if the original producer could profitably set the price low enough to deter unauthorized reproduction. Hence, we will be mainly interested in case (b) henceforth.

The monopolist incurs fixed costs of development, marketing, and promotion. In addition, he incurs the marginal reproduction cost of $c$ for each additional unit of the product. ${ }^{6}$ We assume $c \leq \alpha+z \leq 1$ to have both the legitimate product and the copies co-exist. ${ }^{7}$ Given the consumers' choices, the monopolist chooses the optimal price and quantity by equating his marginal revenue with the marginal cost. The equilibrium outcome for case (b) is characterized as follows. ${ }^{8}$ The equilibrium price, quantity, and profit are given by

\footnotetext{
${ }^{5}$ Note that Figure 1 deals with the case after digitalization. If we set $\alpha^{\prime}=\alpha, s=0, c^{\prime}=c$, and $z^{\prime}=z$, this diagram can also be used for the benchmark case here.

${ }^{6}$ The marginal cost may include marketing and/or promotion costs in addition to the costs directly related to the production and distribution.

${ }^{7}$ If $\alpha+z>1$, then $w \equiv \alpha v+z>v$ for all $v \in[0,1]$. So there exists no unauthorized reproduction. If $c>\alpha+z$, then no consumer buys from the monopolist. See Yoon (2002) for details.

${ }^{8}$ To have an equilibrium outcome in which both the original and copies exist, i.e., of case (b), the parameters need to satisfy $c \geq z-\alpha+2 \alpha z /(1-\alpha)$, or to rearrange, $z \leq(1-\alpha)(\alpha+c) /(1+\alpha)$.
} 


$$
\{p, q, \pi\}=\left\{\frac{\alpha+z+c}{2}, \frac{\alpha+z-c}{2 \alpha}, \frac{(\alpha+z-c)^{2}}{4 \alpha}\right\} .
$$

Consumers who belong to $[0, z /(1-\alpha))$ do not consume the product, consumers who belong to $[z /(1-\alpha),(\alpha+c-z) / 2 \alpha)$ make unauthorized copies, and consumers who belong to $[(\alpha+c-z) / 2 \alpha, 1]$ buy from the monopolist. ${ }^{9}$

The consumer surplus $C S$ and the social welfare $S W$ are given by

$$
\begin{gathered}
C S=\int_{z /(1-\alpha)}^{(\alpha+c-z) / 2 \alpha}(v-\alpha v-z) d v+\int_{(\alpha+c-z) / 2 \alpha}^{1}\left(v-\frac{\alpha+z+c}{2}\right) d v, \\
S W=C S+\pi=\int_{z /(1-\alpha)}^{(\alpha+c-z) / 2 \alpha}(v-\alpha v-z) d v+\int_{(\alpha+c-z) / 2 \alpha}^{1}(v-c) d v .
\end{gathered}
$$

\subsection{The digitalization}

The digitalization of music products affects several aspects of the model. First, the cost structure of music production changes. Let $c^{\prime}$ denote the new marginal cost. Second, the parameters that pertain to unauthorized reproduction may be affected. Let $\alpha^{\prime}$ and $z^{\prime}$ denote the new quality degradation parameter and the consumers' reproduction costs, respectively. Finally, digitalization may enhance the valuation that consumers obtain from legitimate music products. The reason is due to the 'sampling effect' among others: Sampling allows users to experience the music and to find better fits for their tastes. In other words, as Liebowitz (2005) convincingly argues with a candy bar example, sampling in effect provides the users more utility. We want to note that we are not saying here that the actual sales may increase due to sampling, ${ }^{10}$ but only that consumers' valuations may increase because of enhanced satisfaction or reduced risks associated with the purchase. We introduce another parameter $s$ to capture this effect.

We now assume that consumer $i$ gets the net utility of $(1+s) v_{i}-p$ when she buys from the monopolist, with $s \geq 0$. The net utility from a copy is $\left(1-\alpha^{\prime}\right) v_{i}-z^{\prime} \equiv v_{i}-w_{i}{ }^{\prime}$,

\footnotetext{
${ }^{9}$ This is a result in Yoon(2002, Proposition 3(i)). Alternatively, we can apply the analysis in the next subsection by setting $\alpha^{\prime}=\alpha, s=0, c^{\prime}=c$, and $z^{\prime}=z$.

${ }^{10}$ Peitz and Waelbroeck (2006b) show that the music industry may gain from sampling if there is sufficient taste heterogeneity and sufficient product diversity. Our main concern in this paper, however, is different. As a matter of fact, we show below that the sampling effect may sometimes decrease the revenue. See the discussion after Figure 2.
} 
and the net utility from no consumption is normalized again to zero. Consumer choice is determined by the relative magnitudes of $(1+s) v_{i}-p,\left(1-\alpha^{\prime}\right) v_{i}-z^{\prime}$, and zero. Since

$$
\max \left\{(1+s) v-p, v-\left(\alpha^{\prime} v+z^{\prime}\right), 0\right\} \Leftrightarrow \min \left\{p-s v, \alpha^{\prime} v+z^{\prime}, v\right\}
$$

we can determine consumers' choices by using the following diagram.

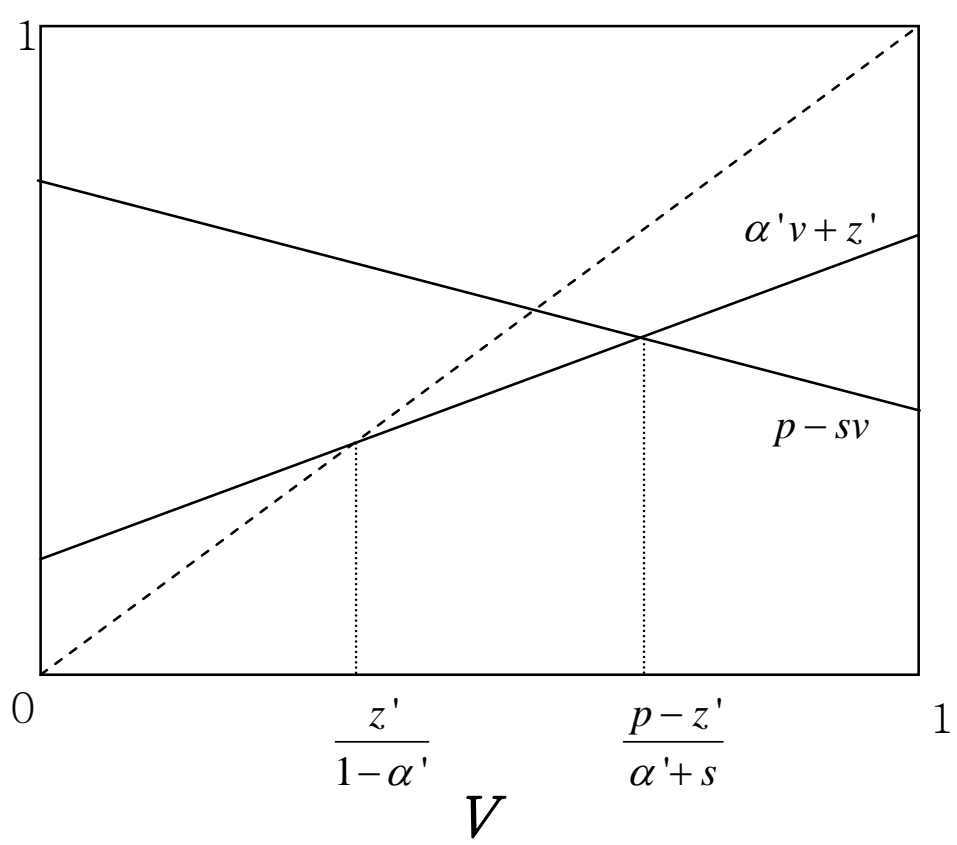

Figure 1: Consumer Choice

Hence, given the monopolist's price $p$, consumers who belong to $\left[0, z^{\prime} /\left(1-\alpha^{\prime}\right)\right)$ do not consume the product, consumers who belong to $\left[z^{\prime} /\left(1-\alpha^{\prime}\right),\left(p-z^{\prime}\right) /\left(\alpha^{\prime}+s\right)\right)$ make unauthorized copies, and consumers who belong to $\left[\left(p-z^{\prime}\right) /\left(\alpha^{\prime}+s\right), 1\right]$ buy from the monopolist.

Note that the diagram is shown for the case when unauthorized copies co-exist with the original. Similar diagram can be drawn for the case when the monopolist charges the price low enough that no consumer makes an unauthorized copy.

The demand function for the legitimate product is therefore

$$
D(p)=\left\{\begin{array}{lll}
1-\frac{p-z^{\prime}}{\alpha^{\prime}+s} & \text { when } & p \geq \frac{(1+s) z^{\prime}}{1-\alpha^{\prime}}, \\
1-\frac{p}{1+s} & \text { when } & p<\frac{(1+s) z^{\prime}}{1-\alpha^{\prime}} .
\end{array}\right.
$$

The marginal revenue function is given as 


$$
M R(q)=\left\{\begin{array}{lll}
\alpha^{\prime}+s+z^{\prime}-2\left(\alpha^{\prime}+s\right) q & \text { when } & q \leq 1-\frac{z^{\prime}}{1-\alpha^{\prime}}, \\
1+s-2(1+s) q & \text { when } & q>1-\frac{z^{\prime}}{1-\alpha^{\prime}} .
\end{array}\right.
$$

By equating this with the marginal cost of $c^{\prime}$ for the case when unauthorized copies co-exist with legitimate products, ${ }^{11}$ we get the equilibrium price, quantity, and profit after digitalization as

$$
\left\{p^{\prime}, q^{\prime}, \pi^{\prime}\right\}=\left\{\frac{\alpha^{\prime}+s+z^{\prime}+c^{\prime}}{2}, \frac{\alpha^{\prime}+s+z^{\prime}-c^{\prime}}{2\left(\alpha^{\prime}+s\right)}, \frac{\left(\alpha^{\prime}+s+z^{\prime}-c^{\prime}\right)^{2}}{4\left(\alpha^{\prime}+s\right)}\right\} .
$$

The consumers' choices can be summarized as follows:

(i) Consumers who belong to $\left[0, z^{\prime} /\left(1-\alpha^{\prime}\right)\right)$ do not consume the product,

(ii) Consumers who belong to $\left[z^{\prime} /\left(1-\alpha^{\prime}\right),\left(\alpha^{\prime}+s+c^{\prime}-z^{\prime}\right) / 2\left(\alpha^{\prime}+s\right)\right)$ make unauthorized copies, and

(iii) Consumers who belong to $\left[\left(\alpha^{\prime}+s+c^{\prime}-z^{\prime}\right) / 2\left(\alpha^{\prime}+s\right), 1\right]$ buy from the monopolist.

The new consumer surplus CS' and the new social welfare $S W^{\prime}$ are

$$
\begin{aligned}
& C S^{\prime}=\int_{z^{\prime}\left(1-\alpha^{\prime}\right)}^{\left(\alpha^{\prime}+s+c^{\prime}-z^{\prime}\right) / 2\left(\alpha^{\prime}+s\right)}\left(v-\alpha^{\prime} v-z^{\prime}\right) d v+\int_{\left(\alpha^{\prime}+s+c^{\prime}-z^{\prime}\right) / 2\left(\alpha^{\prime}+s\right)}^{1}\left((1+s) v-\frac{\alpha^{\prime}+s+z^{\prime}+c^{\prime}}{2}\right) d v, \\
& S W^{\prime}=C S^{\prime}+\pi^{\prime}=\int_{z^{\prime}\left(1-\alpha^{\prime}\right)}^{\left(\alpha^{\prime}+s+c^{\prime}-z^{\prime}\right) / 2\left(\alpha^{\prime}+s\right)}\left(v-\alpha^{\prime} v-z^{\prime}\right) d v+\int_{\left(\alpha^{\prime}+s+c^{\prime}-z^{\prime} / 2\left(\alpha^{\prime}+s\right)\right.}^{1}\left((1+s) v-c^{\prime}\right) d v .
\end{aligned}
$$

\subsection{Comparative statics}

We now turn to some comparative statics. To relate the benchmark to the digitalization, define the functions

$$
p(\alpha, s, c, z)=\frac{\alpha+s+z+c}{2} ; q(\alpha, s, c, z)=\frac{\alpha+s+z-c}{2(\alpha+s)} ; \pi(\alpha, s, c, z)=\frac{(\alpha+s+z-c)^{2}}{4(\alpha+s)} .
$$

Moreover, we have

$$
\begin{aligned}
& C S(\alpha, s, c, z)=\frac{1-\alpha-c-z}{2}+\frac{(\alpha+s+c-z)^{2}}{8(\alpha+s)}+\frac{z^{2}}{2(1-\alpha)}, \\
& S W(\alpha, s, c, z)=C S(\alpha, s, c, z)+\pi(\alpha, s, c, z) .
\end{aligned}
$$

11 For this, the condition $c^{\prime} \geq z^{\prime}-\alpha^{\prime}-s+2\left(\alpha^{\prime}+s\right) z^{\prime} /\left(1-\alpha^{\prime}\right)$, or to rearrange, the condition $z^{\prime} \leq\left(1-\alpha^{\prime}\right)\left(\alpha^{\prime}+c^{\prime}+s\right) /\left(1+\alpha^{\prime}+2 s\right)$ needs to hold. 
Then, the equilibrium prices of the benchmark and after digitalization are $p(\alpha, 0, c, z)$ and $p\left(\alpha^{\prime}, s, c^{\prime}, z^{\prime}\right)$, respectively. We have similar expressions for other variables. We restate two relevant inequality constraints

$$
\begin{gathered}
c \leq \alpha+z \leq 1 \\
c \geq z-\alpha-s+\frac{2(\alpha+s) z}{1-\alpha}
\end{gathered}
$$

that ensure the co-existence equilibrium outcome in which both the legitimate purchase and the unauthorized reproduction prevail.

First, we have

$$
\frac{\partial p}{\partial \alpha}=\frac{\partial p}{\partial s}=\frac{\partial p}{\partial c}=\frac{\partial p}{\partial z}=\frac{1}{2}>0
$$

Hence, the equilibrium price increases as a copy's quality degradation $(\alpha)$, the additional benefits $(s)$, the monopolist's marginal costs $(c)$, and the consumers' reproduction costs $(z)$ increase. Note that the positive effects of $\alpha$ and $z$ are intuitive since the copies are (inferior) substitutes of the original. It is also obvious to see the positive effects of $s$ and $c$.

Next, we have

$$
\frac{\partial q}{\partial \alpha}=\frac{\partial q}{\partial s}=-\frac{z-c}{2(\alpha+s)^{2}} ; \quad \frac{\partial q}{\partial c}=-\frac{1}{2(\alpha+s)}<0 ; \quad \frac{\partial q}{\partial z}=\frac{1}{2(\alpha+s)}>0 .
$$

Hence, an increase in $c$ decreases the equilibrium quantity, while an increase $z$ increases it. The effects of both $\alpha$ and $s$ are the same, and may be positive or negative depending on the relative magnitudes of $c$ and $z$. So, the effects are negative when the consumers' marginal cost is higher than the monopolist's marginal cost, and vice versa. Figure 2 shows the marginal revenue curve $M R(q)=\alpha+s+z-2(\alpha+s) q$. When $\alpha$ or $s$ increases, the curve turns clockwise with the center of $q=1 / 2$ and $M R=z$. Hence, $\partial q / \partial \alpha=\partial q / \partial s>0$ if and only if $c>\mathrm{Z} \cdot{ }^{12}$

\footnotetext{
${ }^{12}$ We note that $\partial q / \partial \alpha=\partial q / \partial s<0$ is possible only when $1-z /(1-\alpha)>1 / 2$ as well as $c<z$.
} 


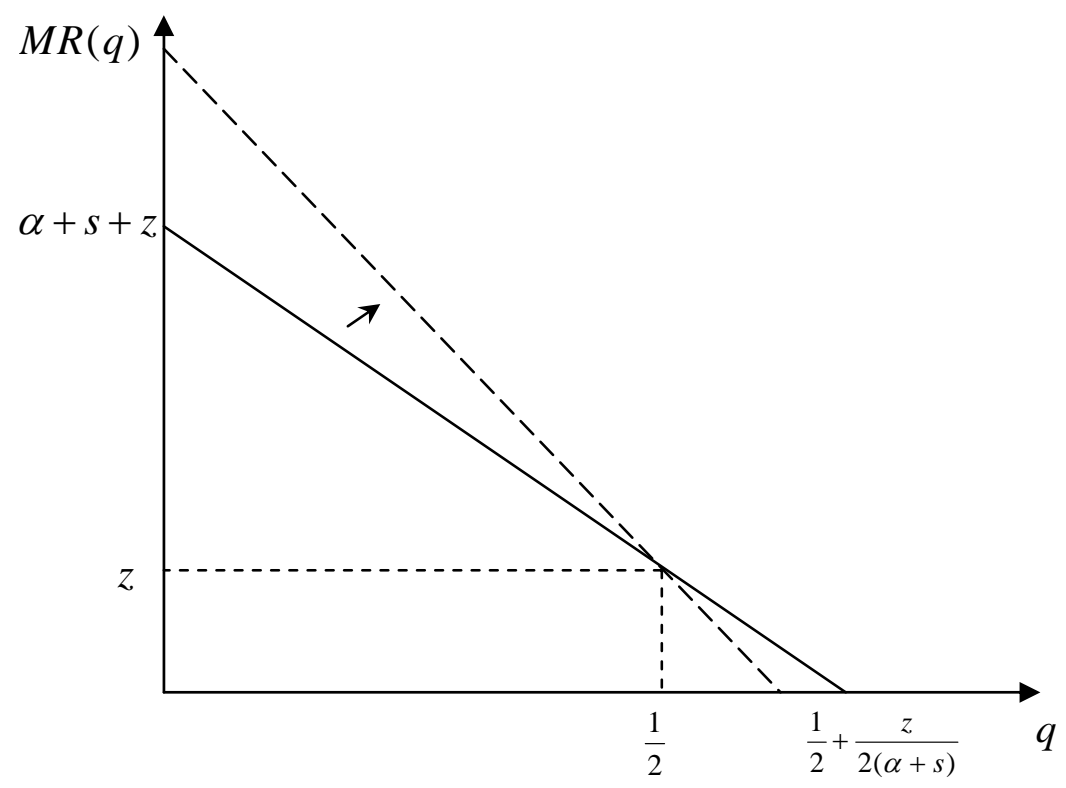

Figure 2: Marginal Revenue

It is worth noting that the revenue $R=p \cdot q$ may increase or decrease when $\alpha$ or $s$ increases. We have

$$
\frac{\partial R}{\partial s}=p \cdot \frac{\partial q}{\partial s}+q \cdot \frac{\partial p}{\partial s}=\frac{1}{4}-\frac{z^{2}-c^{2}}{4(\alpha+s)^{2}} .
$$

It is easy to see that $\partial R / \partial s>0$ when $c \geq z$ (since, in that case, both the quantity and the price increase) and also when $c=0$ by the inequality constraint (2). Though $\partial R / \partial s>0$ for most parameter values, there do exist cases when $\partial R / \partial s<0$. For example, when $(\alpha, s, c, z)=(0.1,0.0,0.1,0.16)$, we have $\partial R / \partial s=-0.0056$. Note that the parameter values satisfy inequality constraint (1) and (2) as well as $c<z$. Note also that the same argument applies to $\alpha$ since $\partial R / \partial \alpha=\partial R / \partial \mathrm{s}$.

Third, we have

$$
\begin{gathered}
\frac{\partial \pi}{\partial \alpha}=\frac{\partial \pi}{\partial s}=\frac{(\alpha+s+c-z)(\alpha+s+z-c)}{4(\alpha+s)^{2}} \geq 0 ; \\
\frac{\partial \pi}{\partial c}=-\frac{\alpha+s+z-c}{2(\alpha+s)} \leq 0 ; \quad \frac{\partial \pi}{\partial z}=\frac{\alpha+s+z-c}{2(\alpha+s)} \geq 0 .
\end{gathered}
$$

Note that $\alpha+s+z-c \geq 0$ by the inequality constraint (1), and $\alpha+s+c-z \geq 0$ by the inequality constraint (2) above. All these effects seem quite intuitive.

Turning to the consumer surplus, we have

$$
\frac{\partial C S}{\partial \alpha}=-\frac{1}{2}\left(1-\frac{\alpha+s+c-z}{2(\alpha+s)}\right)^{2}-\frac{1}{2}\left(\frac{\alpha+s+c-z}{2(\alpha+s)}-\left(\frac{z}{1-\alpha}\right)^{2}\right) \leq 0 ;
$$




$$
\begin{gathered}
\frac{\partial C S}{\partial s}=\frac{(\alpha+s+c-z)(\alpha+s+z-c)}{8(\alpha+s)^{2}} \geq 0 ; \quad \frac{\partial C S}{\partial c}=-\frac{\alpha+s+z-c}{4(\alpha+s)} \leq 0 \\
\frac{\partial C S}{\partial z}=-\frac{1}{2}\left(1+\frac{\alpha+s+c-z}{2(\alpha+s)}\right)+\frac{z}{1-\alpha} \leq-\frac{1}{2}\left(1+\frac{z}{1-\alpha}\right)+\frac{z}{1-\alpha}=-\frac{1}{2}\left(1-\frac{z}{1-\alpha}\right) \leq 0 .
\end{gathered}
$$

These effects are also quite intuitive.

Finally, we can easily see that

$$
\frac{\partial S W}{\partial s}=\frac{3(\alpha+s+c-z)(\alpha+s+z-c)}{8(\alpha+s)^{2}} \geq 0 ; \quad \frac{\partial S W}{\partial c}=-\frac{3(\alpha+s+z-c)}{4(\alpha+s)} \leq 0 .
$$

As for the effect of consumers' reproduction costs, observe that the function

$$
\frac{\partial S W}{\partial z}=-\frac{1}{2}-\frac{\alpha+s+c-z}{4(\alpha+s)}+\frac{z}{1-\alpha}+\frac{\alpha+s+z-c}{2(\alpha+s)}
$$

is a strictly increasing function of $z$ over the interval $[0,(1-\alpha)(\alpha+s+c) /(1+\alpha+2 s)]$. We also find that $\partial S W / \partial z=-(\alpha+s+3 c) /(4(\alpha+s))<0 \quad$ at $\quad z=0 \quad$ and $\partial S W / \partial z=(1+s-c) /(2(1+\alpha+2 s))>0$ at $z=(1-\alpha)(\alpha+s+c) /(1+\alpha+2 s)$. Therefore, the social welfare is a convex function which attains its minimum at

$$
z^{*}=(1-\alpha)(\alpha+s+3 c) /(3+\alpha+4 s) .
$$

The SW strictly decreases over the interval $\left[0, z^{*}\right]$ and strictly increases over the interval $\left[z^{*},(1-\alpha)(\alpha+s+c) /(1+\alpha+2 s)\right]$. See Figure 3 below for representative curves. This behavior of the social welfare is due to two countervailing effects. First, an increase in $z$ directly decreases the social welfare since it increases the reproduction costs of those consumers who choose to copy. Second, an increase in $z$ induces some consumers to switch from making copies to buying from the original producer. This increases the social welfare because this switch decreases the social costs of production: Note that the marginal consumers' reproduction costs who switch are higher than the producer's marginal cost due to the monopoly pricing. ${ }^{13}$

As for the quality degradation, we observe that the function

$$
\frac{\partial S W}{\partial \alpha}=\frac{3(\alpha+s+c-z)(\alpha+s+z-c)}{8(\alpha+s)^{2}}-\frac{1}{2}\left(1-\left(\frac{z}{1-\alpha}\right)^{2}\right)=\frac{\partial S W}{\partial s}-\frac{1}{2}\left(1-\left(\frac{z}{1-\alpha}\right)^{2}\right)
$$

is a strictly increasing function of $\alpha$, so the social welfare is a convex function of $\alpha$. Depending on the relative magnitude of the parameters, the effect of $\alpha$ on $S W$ may

13 For a detailed discussion, see Yoon (2002). 
be positive or negative.

Summarizing the discussion, we have the following table.

$<$ Table $1>$ Comparative statics

\begin{tabular}{|c|c|c|c|c|c|}
\hline & $\partial p$ & $\partial q$ & $\partial \pi$ & $\partial C S$ & $\partial S W$ \\
\hline$\partial \alpha$ & + & + or - & + & - & + or - \\
\hline$\partial s$ & + & + or - & + & + & + \\
\hline$\partial c$ & + & - & - & - & - \\
\hline$\partial z$ & + & + & + & - & - then + \\
\hline
\end{tabular}

\section{Main results}

\subsection{The impacts of digitalization}

As discussed earlier, the digitalization of music products affects the equilibrium outcome through various routes: The quality of unauthorized copies may change ( $\alpha$ to $\alpha^{\prime}$ ), consumers may get additional benefits ( $\left.s \geq 0\right)$, the original producer's marginal cost may change ( $c$ to $c^{\prime}$ ), and the consumers' reproduction costs may change ( $z$ to $\left.z^{\prime}\right)$. Therefore, it is a difficult task to measure the net impacts of digitalization. Nevertheless, we will try to calibrate the model using some empirical observations about the price and the quantity, and determine the impacts of digitalization on the profit, the consumer surplus, and the social welfare. We are fully aware that this exercise is only heuristic at the best, and more empirical works need to be accumulated to accurately pin down the actual effects of digitalization.

One of the most obvious empirical facts after digitalization is the decline in the sales of recorded music. Liebowitz (2006), for example, estimates that the actual sales in the United States have dropped up to $30 \%$ after digitalization. ${ }^{14}$ While various explanations may be possible to account for this decrease, ${ }^{15}$ most of the existing empirical works attribute the major cause to the file sharing activities made possible by the digitalization. For example, Peitz and Waelbroeck (2004) find that music downloading could have caused a 20\% reduction in music sales worldwide between 1998-2002. Michel (2006)

\footnotetext{
${ }^{14}$ See Figure 3 of Liebowitz (2006). He uses the RIAA (Recording Industry Association of America) data on unit quantities of full-length albums.

15 Liebowitz (2005), for example, considers other factors such as income changes, changes in substitute/complement markets, changes in the quality of music, and changes in the supply of music for possible explanations.
} 
finds that some music consumers could have decreased their CD purchases (prior to 2004) by about $13 \%$ due to Internet file sharing. Zentner (2006) finds that music downloading reduces the probability of buying music by 30\%, and sales in 2002 would have been around $7.8 \%$ higher without downloads. ${ }^{16}$ This is plausible since the unauthorized copies easily obtainable through the Internet directly compete with the legitimate copies.

Another interesting and rather puzzling empirical fact after digitalization is the virtual constancy of the album prices, especially in the United States. Liebowitz (2006) observes that 'the list prices adjusted for inflation have been virtually constant for the last decade,' and Peitz and Waelbroeck (2005) provide similar observations. ${ }^{17}$ If we admit that the unauthorized copies work as substitutes for the legitimate copies, we naturally expect that the original producer's price should be lower after the file sharing activities. But, we observe otherwise. Why is this happening?

To see the reason, let us fit our model to these empirical observations. We make the following assumptions on the parameter changes. First, the move to online distribution channel seems to reduce the marginal costs of reproduction and distribution. In addition, the marketing and promotion costs would probably decline. It is thus fair to assume that the new marginal cost $c^{\prime}$ is not higher than the original marginal cost $c$, that is, $c^{\prime} \leq c$. Second, the quality degradation parameter may not increase due to digitalization, that is to say, digitalization may not deteriorate the quality of unauthorized copies. So, assume $\alpha^{\prime} \leq \alpha$. Third, the consumers' reproduction costs may decrease due to easier access (say, via P2P networks) and copying technology. So, assume $z^{\prime} \leq z$. Finally, we already set $s \geq 0$ to reflect the sampling effect.

Given the comparative static analysis of the previous section, it is not hard to see that consumers get substantial additional benefits from the digitalization. The observed price has remained virtually constant while the changes in $\alpha, c$, and $\mathrm{Z}$ after digitalization should have lowered it. Hence, the increase in $s>0$ must have counteracted against the price decrease. On the other hand, the cause for the observed decrease in quantity may have come from several directions: The decreased reproduction costs $Z$ have stolen significant parts of the legitimate demand, and the monopolist may have reacted to the additional benefit factor $s$ by focusing more on the high valuation consumers. ${ }^{18}$ It is noteworthy that the consumer surplus has increased undoubtedly after the

\footnotetext{
${ }^{16}$ On the other hand, Oberholzer and Strumpf (2005) find that file sharing has only had a limited effect on record sales.

${ }^{17}$ See p. 21 as will as footnote 30 of Liebowitz (2006), and Figures 4 and 5 of Peitz and Waelbroeck (2005).

${ }^{18}$ Recall that the optimal quantity decreases as $S$ increases when $c<z$.
} 
digitalization, while the profit and the social welfare may have increased or decreased.

We now further specify the model to investigate the possible impacts of digitalization in depth. Let us assume that $c=c^{\prime}=0$, as many previous papers on the copyright issues do. ${ }^{19}$ That is, we assume that the marginal reproduction costs of the legitimate copies are negligible. Casual observation seems to support this normalization since the variable costs are quite low compared to the album price. Let us also assume $\alpha=\alpha^{\prime}$, that is, the quality degradation parameter remains constant. Note that the sound quality of MP3 music files shared on the P2P networks is generally perceived to be inferior to the sound quality of the original soundtracks of CDs due to the compression rates. Observe also that the quality degradation may result from the lack of accompanying lyrics, the lack of photographic illustrations of the singers, and the lack of technical supports. It is therefore reasonable to assume that the quality degradation parameter pertaining to unauthorized copies did not change significantly even with the advent of online distribution. On the other hand, we capture the effects of digitalization on unauthorized copies with the parameter $z$. We assume $z^{\prime}<z$, because the digital distribution channel made it easier for consumers to access and copy the music files.

With these additional assumptions, the price and quantity changes after digitalization are

$$
p=\frac{\alpha+z}{2} \Rightarrow p^{\prime}=\frac{\alpha+s+z^{\prime}}{2} ; q=\frac{\alpha+z}{2 \alpha} \Rightarrow q^{\prime}=\frac{\alpha+s+z^{\prime}}{2(\alpha+s)},
$$

and we have

$$
\frac{p^{\prime}}{p}=\frac{\alpha+s+z^{\prime}}{\alpha+z} \text { and } \frac{q^{\prime}}{q}=\frac{\alpha}{\alpha+s} \cdot \frac{\alpha+s+z^{\prime}}{\alpha+z}=\frac{\alpha}{\alpha+s} \cdot \frac{p^{\prime}}{p} .
$$

The empirical fact of constant price implies that $z^{\prime}+s=z$. Moreover, the actual quantity decrease due to the digitalization is $100 x \%$ so that $q^{\prime} / q=1-x$, we get

$$
s=\frac{x}{1-x} \alpha \text {. }
$$

Hence, regarding the impact on the price, the additional benefits factor has just offset the decrease in the consumers' reproduction costs, and that factor is about $43 \%$ (25\%, $11 \%$, respectively) of the quality degradation parameter when the quantity decreases by $30 \%$ (20\%, 10\%, respectively). ${ }^{20}$ Note that $s$ has a positive relationship with $x$.

The change in the profit after digitalization is given by

\footnotetext{
19 See, for example, Belleflamme (2003) and Bae and Choi (2006).

${ }^{20}$ We mention that other factors that may affect the quantity decline work to change the price either way. Hence, price constancy may be sustained regardless of the actual quantity change due to the digitalization.
} 


$$
\frac{\pi^{\prime}}{\pi}=\frac{\left(\alpha+s+z^{\prime}\right)^{2}}{\alpha+s} \cdot \frac{\alpha}{(\alpha+z)^{2}}=\frac{\alpha}{\alpha+s} \cdot\left(\frac{p^{\prime}}{p}\right)^{2}=1-x .
$$

The change in the profit is equivalent to the change in the quantity since the marginal cost is assumed to be zero and the actual price did not change. Hence, the profit has decreased by $100 x \%$. As Liebowitz (2006, p. 18) argues, the sampling effect (the increase in $s$ ) could not 'counterbalance the negative impacts of the substitution effect' (the decrease in $z$ ). We want to add, however, that the present model does not consider the possible changes in fixed costs. It is generally expected that the digitalization may substantially lower the distribution costs. In particular, sampling may replace costly marketing and promotion activities. Moreover, the file sharing activity may also increase the additional revenue from complementary products such as live performance by broadening the audiences. See Gayer and Shy (2006) for a related discussion. It is therefore possible that the profit has not decreased so much as predicted in this simple model. On the other hand, we have $\Delta C S>0$, as discussed earlier, since $\partial C S / \partial s>0$ and $\partial C S / \partial z<0$. So, the consumer surplus has increased.

To see the impact on the social welfare, let us define $\Delta s=s-0=s$ and $\Delta z=z-z^{\prime}$. Note that $\Delta s$ and $\Delta z$ are positive, and both are equal to $x \alpha /(1-x)$. Then, the change in the social welfare is ${ }^{21}$

$$
\begin{aligned}
\Delta S W & =\frac{3(\alpha+z)(\alpha-z)}{8 \alpha^{2}} \cdot \Delta s+\left(\frac{1}{2}+\frac{\alpha-z}{4 \alpha}-\frac{z}{1-\alpha}-\frac{\alpha+z}{2 \alpha}\right) \cdot \Delta z \\
& =\frac{5 x}{8(1-x)} \alpha-\left(\frac{1}{1-\alpha}+\frac{3}{4 \alpha}\right) \frac{x}{1-x} \alpha z-\frac{3 x}{8(1-x) \alpha} z^{2} .
\end{aligned}
$$

Since $\Delta S W=5 \alpha x / 8(1-x)>0$ at $z=0$ and $\Delta S W=-3 x / 8 \alpha(1-x)<0$ at $z=1-\alpha$, we conclude that the social welfare has increased after digitalization if and only if $z$ is small. The reason is that the social welfare is a decreasing function of $z$ for small $z<z^{*} \equiv(1-\alpha)(\alpha+s) /(3+\alpha+4 s)$ and an increasing function of $z$ for large $z>z^{*}$.

The following table shows numerical calculations for some parameter values. The numbers in the cells of columns 2-4 are the cut-off values of $z$ corresponding to respective $\alpha$ and $x$. For example, when $\alpha=0.2$ and $x=0.3$, the cut-off is 0.161 . The social welfare increases for the initial values of $z$ that are smaller than the cut-off, while it decreases for the values of $z$ that exceed the cut-off. The last column shows the upper bound for $z$ given by inequality constraint (2) above. That is, only the

\footnotetext{
${ }^{21}$ We measure the impact at the initial point, that is, prior to the digitalization by setting $s=0$. We get the same qualitative conclusion when we measure the impact at the final point, that is, posterior to the digitalization by setting $s=x \alpha /(1-x)$.
} 
values of $z$ below the upper bound are relevant.

$<$ Table 2> The upper bound and the cut-off values of $Z$

\begin{tabular}{|c|c|c|c|c|}
\hline & $x=0.1$ & $x=0.2$ & $x=0.3$ & $\begin{array}{c}\text { upper bound } \\
\text { for } z\end{array}$ \\
\hline$\alpha=0.05$ & 0.034 & 0.040 & 0.046 & 0.045 \\
\hline$\alpha=0.1$ & 0.066 & 0.076 & 0.088 & 0.082 \\
\hline$\alpha=0.2$ & 0.120 & 0.138 & 0.161 & 0.133 \\
\hline$\alpha=0.3$ & 0.160 & 0.186 & 0.218 & 0.162 \\
\hline$\alpha=0.4$ & 0.187 & 0.219 & 0.259 & 0.171 \\
\hline$\alpha=0.5$ & 0.198 & 0.237 & 0.284 & 0.166 \\
\hline
\end{tabular}

Note: The cut-off values are found numerically by setting the difference in social welfare functions $S W(\alpha, \alpha x /(1-x), 0, z-\alpha x /(1-x))-S W(\alpha, 0,0, z)$ to zero.

Note that the cut-off increases as $\alpha$ and $x$ increase. That is, the social welfare is more likely to increase when the quality degradation is higher and/or the actual sales decline is higher. Table 2 reveals that the social welfare increases for most values of the parameters. In particular, the social welfare increases unambiguously in the case of a $30 \%$ quantity decline since the cut-off is always higher than the upper bound. The social welfare also increases unambiguously for parameter values of $\alpha$ exceeding 0.4 .

In summary, empirical facts support the conclusion that (i) the profit has decreased, (ii) the consumer surplus has increased, and (iii) the social welfare has either increased or decreased due to the digitalization. The social welfare has increased if and only if the consumers' reproduction costs have been relatively low, and numerical calculations reveal that it has increased for most parameter values.

\subsection{The effect of further protection}

The empirical observations together with our model support the conclusion that firms' profits have declined due to digital music distribution. This is so even when the additional benefits factor $s$ may have contributed positively to the profits. Hence, the firms' incentive to develop valuable new products may not be sufficiently provided since the profits may not cover the development and other costs. Addressing this problem, it is often argued that further protection of legitimate copies is desirable. This protection is either legal protection such as strengthened copyright laws and enforcement or technological protection such as use of digital rights management 
technology. ${ }^{22}$

It is a very difficult task to discuss the trade-off between dynamic efficiency and static efficiency, that is, to meaningfully compare the development phase and the usage phase of the copyrightable works and obtain testable conclusions on the relevant economic variables. We do not attempt to do this in this paper. Instead, while fully appreciating the importance of the dynamic incentive to create, we just want to point out the fact that 'reversing the substitution effect' by increasing $z$ with legal and/or technological measures may have some unwanted effects on the social welfare. One may reason that this reversal will also reverse the impact of $z$ on the social welfare. So, if the effect of $z$ on the social welfare has been negative, then the stronger copyright or the digital rights management technology would increase the social welfare. This is not true.

Figure 3 depicts two social welfare curves as functions of $z$. The curve $S W_{1}$ corresponds to the benchmark before the digitalization, while the curve $S W_{2}$ corresponds to the situation after the digitalization. Since $s$ has increased, $S W_{2}$ lies above $S W_{1}$. Observe also that $z_{1}^{*}$, the minimum point of $S W_{1}$, lies to the left of $z_{2}^{*}$, the minimum point of $S W_{2}$.

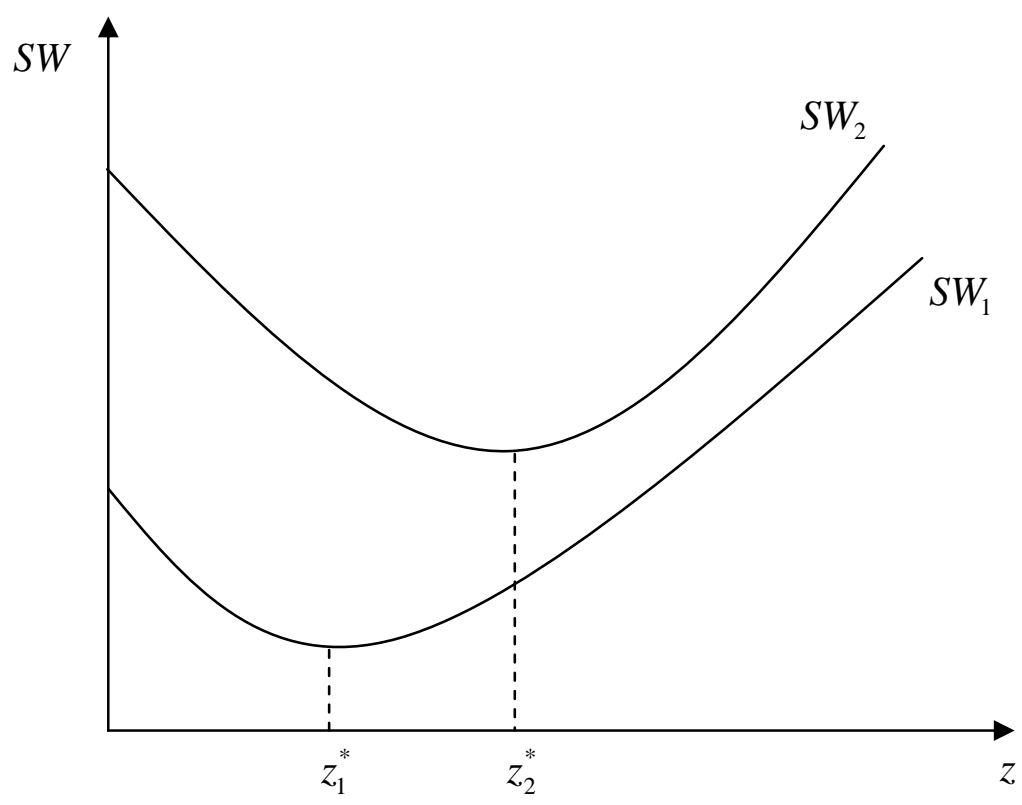

\footnotetext{
${ }^{22}$ The DRM, short for the digital rights management, technology can be defined generally as the 'secure packaging and delivery software designed to prevent purchasers and third parties from making unauthorized uses of digital works.' The DRM is a small piece of software that can detect, monitor and block use of copyrighted material. The DRM for music generally includes: copy control, watermarking, fingerprinting, authentication and access control.
} 
Figure 3: The social welfare

Suppose that the initial reproduction cost was relatively high, say somewhat bigger than $z_{1}^{*}$. Then, the digitalization that has lowered $z$ would have a negative impact on the social welfare along the curve $S W_{1}$. An increase in $z$ after digitalization, however, will change the social welfare along the curve $S W_{2}$. As long as this increase does not go beyond $z_{2}^{*}$, we see that the effect on the social welfare is negative again, further decreasing the social welfare. If, on the other hand, the effect of $z$ on the social welfare has been positive, an increased protection would decrease the social welfare. But, this is not desirable at least from the static perspective.

\section{Conclusion}

There have been lively debates regarding the impact of the file sharing technology on the music industry. Most of the arguments, however, are concerned with the causes of the sales decline and its possible consequences on music producers. In this paper, we took it one step further and studied the impacts on such important economic variables as the profit, the consumer surplus, and the social welfare.

We constructed a representative model that enabled the comparative static analysis. We then fitted the model to the empirical observations of substantial sales decline and constant price. Main findings are as follows. First, though the producers' profits have probably shrunk, the decrease may not be as severe as the observed sales decline. Second, the consumer surplus has unambiguously increased. Third, the social welfare may have increased or decreased. It has increased if and only if the consumers' reproduction costs have been relatively low, and numerical calculations reveal that it has increased for most parameter values.

We are fully aware that these conclusions are only suggestive. In particular, though we have tried to make the assumptions on the model specification as reasonable as possible, they still need to be justified by substantial empirical endeavor. We hope that this paper provides a stepping stone for future empirical works. 


\section{References}

Bae, S. and Choi. J. (2006), “A model of piracy,” Information Economics and Policy 18, pp. 303-320.

Belleflamme, P. (2003), "Pricing information goods in the presence of copying," in Gordon, W., Watt, R. (Eds.), The Economics of Copyright: Developments in Research and Analysis, Edward Elgar.

Gayer, A. and Shy, O. (2006), "Publishers, artists, and copyright enforcement," Information Economics and Policy 18, pp. 374-384.

Liebowitz, S. (2005), "Pitfalls in measuring the impact of file-sharing," CESifo Economic Studies 51, pp. 435-473.

Liebowitz, S. (2006), "File sharing: Creative destruction or just plain destruction?" Journal of Law and Economics 49, pp. 1-28.

Michel, N. (2006), "The impact of digital file sharing on the music industry: An empirical analysis,” Topics in Economic Analysis \& Policy 6, article 18.

Oberholzer, F. and Strumpf, K. (2005), “The effect of file sharing on record sales: An empirical analysis,” University of North Carolina working paper.

Peitz, M. and Waelbroeck, P. (2004), “The effect of Internet piracy on CD sales - cross section evidence,” Review of the Economic Research on Copyright Issues 1, pp. 71-79.

Peitz, M. and Waelbroeck, M. (2005), “An economist’s guide to digital music,” CESifo Economic Studies 51, pp. 359-428.

Peitz, M. and Waelbroeck, P. (2006a), "Piracy of digital products: A critical review of the theoretical literature,” Information Economics and Policy 18, pp. 449-476.

Peitz, M. and Waelbroeck, P. (2006b), "Why the music industry may gain from free downloading - The role of sampling," International Journal of Industrial Organization 24, pp. 907-913. 
Yoon, K. (2002), “The optimal level of copyright production,” Information Economics and Policy 14, pp. 347-348.

Zentner, A. (2006), "Measuring the effect of file sharing on music purchases," Journal of Law and Economics 49, pp. 63-90. 